\title{
Utopía como imaginación organizacional en el pensamiento crítico de Guerreiro-Ramos
}

\author{
Utopia as organizational imagination in Guerreiro-Ramos' critical thought \\ Utopia como imaginação organizacional no pensamento crítico do Guerreiro-Ramos
}

\author{
Francisco Salgado ${ }^{1}$ \\ Universidad del Azuay / Instituto de Estudios de Régimen Seccional del Ecuador, Ecuador \\ Escuela Politécnica Nacional / Facultad de Ciencias Administrativas, Ecuador
}

Andrés Abad ${ }^{2}$

Escuela Politécnica Nacional / Facultad de Ciencias Administrativas, Ecuador Universidad Andina Simón Bolívar, Ecuador

\begin{abstract}
Resumen
Este artículo analiza la esencia de la obra de Alberto Guerreiro-Ramos en el marco del pensamiento crítico latinoamericano en los estudios de la administración. Se analizan sus propuestas básicas desde la perspectiva de la utopía, comprendida como una investigación sistemática de principios alternativos de organización distintos a la forma empresarial funcionalista. Se exploran conceptos como de la reducción sociológica, el hombre parentético y la teoría de la delimitación de los sistemas sociales desde un enfoque de las perspectivas utópicas; esto es, la reformulación de las estructuras y dinámicas sociales propuestas por Guerreiro-Ramos como alternativas al ethos del mercado. Asimismo se explica cómo la dinámica y acción recíproca entre la sociedad y los ámbitos organizacionales permiten imaginar alternativas para la emancipación, la búsqueda de la razón sustantiva y la realización plena del ser humano.
\end{abstract}

Palabras claves: Pensamiento Crítico. Teorías Organizacionales. Utopía. Pensamiento Latinoamericano. Guerreiro Ramos.

\begin{abstract}
This article discusses the main issues of the academic production of Alberto Guerreiro-Ramos as part of Latin American critical thought in administration studies. His basic proposals are analyzed from the perspective of utopianism, which is comprehended as a systematic investigation of alternative principles of organization, which are distinctive of the functionalist form of the enterprise. Seminal concepts, such as sociological reduction, parenthetical man and the theory of social systems delimitation, are explored from an utopian perspective approach; i.e. on the reformulation of social structures and dynamics proposed by Guerreiro-Ramos as alternatives to the market ethos. This paper also explains how the dynamic interaction between organizations and society can imagine alternatives to instrumental conceptions of management, options that pursue emancipation, substantive reason and human fulfillment.
\end{abstract}

Keywords: Critical Thought. Organizational Theories. Utopia. Latin American Thought. Guerreiro Ramos.

Artículo enviado en 20 de enero de 2014 y aprobado para publicación en 26 septiembre 2014.

\section{DOI: http://dx.doi.org/10.1590/1679-395115335}

${ }^{1}$ Doctor en Administración por la Universidad Andina Simón Bolívar, Sede Ecuador; Profesor principal del Instituto de Estudios de Régimen Seccional del Ecuador (IERSE) de la Universidad del Azuay; Profesor invitado de la Facultad de Ciencias Administrativas de la Escuela Politécnica Nacional. Dirección: Av. 24 de mayo 777, Cuenca - Ecuador. E-mail: Francisco.Salgado@fulbrightmail.org

2 Doctorando en Administración por la Universidad Andina Simón Bolívar, Sede Ecuador; Profesor titular de la Facultad de Ciencias Administrativas de la Escuela Politécnica Nacional; Profesor invitado del Area de Letras de la Universidad Andina Simón Bolívar, Sede Ecuador. E-mail: andres.abad@uasb.edu.ec 


\section{Resumo}

Este artigo discute a essência da obra de Alberto Guerreiro Ramos-como parte do pensamento crítico latino-americano em estudos de administração. Suas propostas básicas são analisados sob a perspectiva da utopia, que é compreendida como uma investigação sistemática de princípios alternativos de organização, que são distintas da forma funcionalista da empresa. Conceitos seminais como a redução sociológica, homem parentético e a teoria da delimitação dos sistemas sociais, são exploradas a partir de uma abordagem utópica; ou seja, a reformulação das estruturas e dinâmicas sociais propostas por Guerreiro Ramos-como alternativas para o ethos do mercado. Este documento também explica como a interação dinâmica entre as organizações e a sociedade pode imaginar alternativas para concepções instrumentais de gestão; opções que buscam a emancipação, a razão substantiva e a realização humana.

Palavras-chave: Pensamento Crítico. Teorias Organizacionais. Utopia. Pensamento Latino-Americano. Guerreiro Ramos.

\section{Introducción}

Este artículo analiza la obra de Alberto Guerreiro Ramos en el marco del pensamiento crítico latinoamericano. El ensayo aquí propuesto forma parte de una línea de investigación sobre teorías que avizoran alternativas sociales y organizacionales en una dialéctica histórica entre la modernidad y la posmodernidad desde un locus de enunciación latinoamericano. Esta dialéctica permite establecer una mirada de los fenómenos organizacionales desde una perspectiva crítica, toda vez que se parte de una concepción en la que el tiempo actual se encuentra en una emergente etapa de reconfiguración histórica, bien en una modernidad como proyecto inconcluso (HABERMAS, 1989), que implica la apropiación crítica del proyecto moderno para el rescate del sentido emancipador de la razón, o bien en una condición posmoderna que da un sentido de culminación de las metanarrativas y grandes relatos de la modernidad (LYOTARD, 1984).

Esta mirada puede comprenderse como un paradigma general contracorriente en los estudios organizacionales y se basa, primordialmente, en los aportes de la teoría crítica y el posestructuralismo o posmodernismo (ALVESSON y WILLMOTT, 1992). Entonces, para emprender estudios y análisis organizacionales que puedan partir desde una mirada no hegemónica en la administración, es decir, al margen de la corriente principal modernista o mainstream funcionalista, se precisa analizar los referentes teóricos señalados dentro de su diversidad y que además consoliden la praxis como punto de partida, para establecer una mirada sólida e iniciar la comprensión de los fenómenos que se suceden dentro de la organización; en esta perspectiva sociocrítica se inserta el pensamiento de Guerreiro Ramos.

En este contexto, el pensamiento crítico de Guerreiro Ramos y su "Nueva Ciencia" organizacional son analizados desde sus conceptos seminales como la reducción sociológica, el hombre parentético y la teoría de la delimitación de los sistemas sociales, desde los conceptos de la utopía y de la ciencia. La profundización de estas nociones se mira desde varias facetas y ángulos, que demarcan la vasta obra de este pensador brasileño, quien fue influido también por la Escuela de Fráncfort (ANDREWS, 2000) y por lo cual lo identificamos como dentro de la corriente del pensamiento crítico; sin dejar de señalar que otros trabajos, como el de Faria (2009), discuten esta caracterización señalando que la concepción de Guerreiro Ramos no se inscribe en la teoría crítica sino en el idealismo fenomenológico de Husserl.

La propuesta de Guerreiro Ramos tiene, en efecto, un fuerte influjo de la fenomenología de Husserl (1999) y su propio concepto de reducción fenomenológica: el conocimiento de las esencias a través de la transición de una actitud ingenua a una actitud crítica ${ }^{3}$. "La reducción sociológica es una actitud metodológica que busca aprehender las estructuras fundamentales de los conceptos sociológicos, los fenómenos o los datos, mediante

${ }^{3}$ La reducción fenomenológica husserliana, según Schmitt (1959), implica un cambio de actitud hacia un pensamiento crítico, a través de poner entre paréntesis y en suspensión lo que se da por hecho, para reflexionar sobre las esencias subyacentes. 
la reducción o la destilación de los presupuestos referenciales, de carácter histórico, de los hechos u objetos de la realidad" (GUERREIRO RAMOS, 1958, p. 20). También en la concepción del hombre parentético hay una fuerte influencia de Husserl (1999), quien planteó que el conocimiento de las esencias sólo es posible a través de la "parentización", esto es, poner entre paréntesis lo que se supone como ya sabido para llegar a las esencias de las cosas.

La delimitación que Guerreiro Ramos propone, es un intento sistemático para superar el continuo proceso predominante de uniformizar tanto al ser humano como a la vida colectiva. La unidimensionalización es el proceso específico de socialización por el cual el ser humano internaliza profundamente el ethos del mercado y actúa de manera tal como si este ethos fuera la dimensión normativa absoluta del espectro total - el único color del arco iris - de las relaciones interpersonales. Del mismo modo, se pone en evidencia que el campo organizacional es una trama compleja y compuesta por varias dimensiones, especialmente constituida por las relaciones entre los sujetos que lo conforman y que demanda una mayor comprensión de los fenómenos en las organizaciones en la que se desenvuelven y actúan estos individuos, en contraste con la visión meramente instrumental que se centra el mercado y a la maximización de la eficiencia y la productividad. Guerreiro Ramos establece un modelo de razón substantiva, para satisfacer las necesidades de la realización personal con los escenarios sociales y organizacionales, como un pensamiento que apenas comienza a ser descubierto por los estudios sobre la gestión en América Latina.

\section{Discusión}

\section{Perspectivas en los estudios de la gestión}

Las vertientes teóricas crítica y posmodernista son miradas alternativas a la hegemonía funcionalista en la administración (KAYTAL, 2009; VIEIRA y CALDAS, 2006); y, según Alvesson, Bridgman y Willmott (2001), se incorporan dentro de una línea llamada crítica, aunque en muchos aspectos teóricos y epistemológicos son antitéticas. Son perspectivas con la que se puede intentar una reorientación de la corriente instrumental predominante en los estudios en administración. Alvesson y Deetz (2005) idearon una matriz (ver figura 1. infra), que guarda analogía con la que propusieron Burrell y Morgan (1979), en relación con las posturas en la investigación organizacional, en la que se puede observar que el la teoría crítica y el posmodernismo son similares en la medida que contrastan sus perspectivas de "disenso" con los demás enfoques, explicitados en el eje vertical como "relación con el discurso dominante"; pero, a su vez, son diferentes entre sí en la forma de abordar los conceptos y los problemas de investigación.

En el eje horizontal se nota que el posmodernismo contrasta su enfoque en lo "local/ emergente" con las concepciones de la mirada en la "élite/ a priori" de la teoría crítica, que se convierte en una manera "interesante de pensar acerca de las diferencia entre los discursos posmodernistas y de la teoría crítica" desde una mirada "metateórica"; y los autores aclaran que no tratan de "identificar paradigmas sino discursos particulares" (ALVESSON y DEETZ, 2005, p. 68-69). 
Figura 1

Discursos en la investigación organizacional

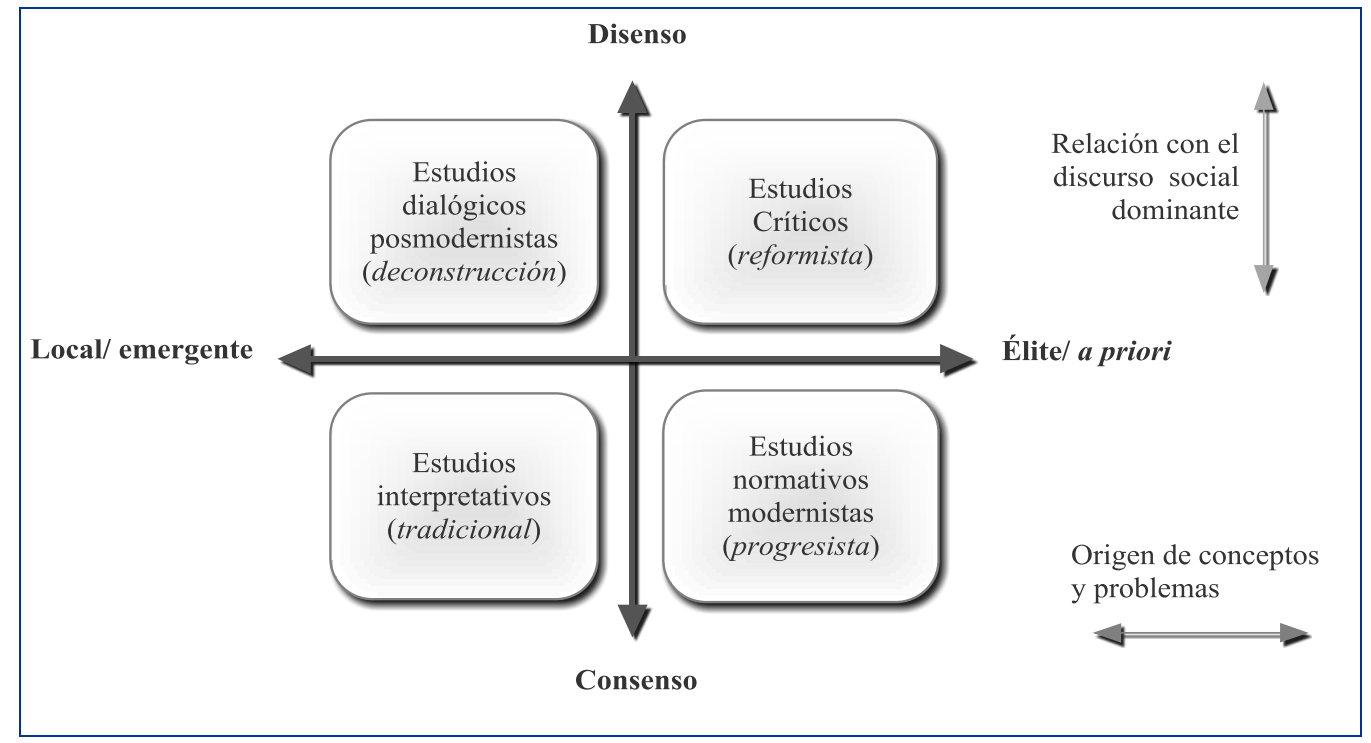

Fuente: Adaptado de Alvesson y Deetz (2005).

La interpretación de esta matriz permite contrastar los dos ejes en posiciones divergentes, por ejemplo, en el eje vertical: si el consenso (c) significa una confianza en la investigación, el disenso (d) es la sospecha; la integración y la armonía son posibles (c), en contraste con la dominación y el conflicto (d); la metáfora epistemológica dominante es el "espejo que refleja" (c), se opone a una metáfora del lente que "mira y lee" (d); la ciencia es "neutral" (c) frente a la ciencia que es "política" (d); el investigador es anónimo y fuera del espacio tiempo que descubre (c) en oposición al investigador históricamente situado que se posiciona (d).

En el eje horizontal que mira una dicotomía entre lo local/ emergente (l) y la élite/ a priori (e): si se estudia la comparación de las comunidades (l), contrasta con el estudio de una comunidad privilegiada (e); los estudios particulares (l) en oposición a lo universal (e); si en el uno hay narrativas locales (l), en el otro grandes narrativas y emancipación (e); la primera mira lo extraño (l) el segundo lo familiar (e); si el uno mira lo sensual y el sentido (l), el otro la racionalidad y la verdad.

La formación de la disciplina administrativa surgió a finales del siglo XIX, con clara meta de ejercer estrategias para el mejoramiento de la eficiencia y productividad; y evidentemente, no nació con una reflexión filosófica centrada en el desarrollo y la emancipación del ser humano en los entornos laborales. Por tanto, desde su génesis, la gestión o administración se consolidó como una serie de recetas de carácter instrumental para constituirse en un soporte a la cadena productiva que estuviera directamente relacionada con el incremento de la rentabilidad. La reflexión, por el contrario, requiere de un proceso de exégesis.

La administración y la gerencia en el mundo académico han seguido fiel a los imaginarios que se han creado en torno a la "eficiencia" de los estudiantes de $\operatorname{los} M B A$, que se orientan a dotar a los directivos de habilidades instrumentales, y en cuya formación los discursos heterodoxos y críticos se quedan en buenas intenciones, casos excepcionales se dan en la formación académica en instituciones que mantienen programas o líneas de investigación crítica, como la del grupo en "Humanismo y Gestión" de la escuela de gestión $H E C$, afiliada a la Universidad de Montreal, que parte de una postura humanista radical, inspirado en el pensamiento de Omar Aktouf (2001) y Jean-François Chanlat (1998).

Perteneciente a esta corriente intelectual es el caso del grupo "Nuevo Pensamiento Administrativo" de la Universidad del Valle en Cali, que trabaja el concepto de las prácticas inhumanas en el trabajo. En esa misma línea, se ubica el "Laboratorio de Investigación en Estrategia, Estructura y Decisión" de la 
Universidad Nacional de Colombia, y el "Grupo de Investigación en Perdurabilidad Empresarial" de la Universidad del Rosario de Colombia, que enfatiza los temas sobre el pensamiento complejo (SAAVEDRA MAYORGA, 2009). Adicionalmente, se debe mencionar la línea que dejó trazada el investigador Eduardo Ibarra Colado (1957-2013), de la Universidad Autónoma Metropolitana-Xochimilco, en México.

Se podría complementar indicando que en América Latina esta visión crítica se encuentra liderada por algunos profesores dentro de las escuela de administración en la Fundación Getulio Vargas, en la Escola Brasileira de Administração Pública e de Empresas (EBAPE), en Río de Janeiro; y en los grupos de investigación en línea crítica de la Universidade Federal de Santa Catarina, de la Universidade Federal do Rio de Janeiro y de la Universidade Federal do Rio Grande do Sul, por ejemplo, en Brasil, sin constituir una lista exhaustiva. Esta tendencia de estudios críticos de la gestión es seguida por el grupo de académicos de la Universidad Andina "Simón Bolívar", sede Ecuador, con su taller de estudios de la gestión, en la que consta una línea crítica en los estudios organizacionales.

Tanto la escuela brasileña como la naciente ecuatoriana tienen influencia del sociólogo brasileño Alberto Guerreiro Ramos, quien escribió varias obras sobre el tema organizacional inspirado, en parte, en los filósofos de la teoría crítica; la reflexión metodológica de este autor es su reducción sociológica, que dice que los problemas de una sociedad particular se explican por la fase en que tal sociedad se encuentra; y el de la ley del carácter subsidiario de la producción científica que expresa que toda producción científica extranjera es en principio auxiliar, o sea, no es un modelo o paradigma, sino un apoyo para la producción del conocimiento local (GUERREIRO RAMOS, 1965). Es en este marco que trabajamos este ensayo para destacar la perspectiva crítica en administración que surge desde un locus de enunciación y de praxis latinoamericanos.

La perspectiva crítica en la administración invita a tener una mirada doble, afirma Tenório (2010), pues mientras más se acerca uno a su estudio, más se aleja del objeto; es decir, la profundización de los conocimientos implica que hay mayor complejidad cuando se quiere cambiar la perspectiva tradicional o desde la visión de los dominadores a los dominados. En otras palabras, cuanto más se trata de concebir la administración como área de conocimiento aplicado, más se aleja uno de ella al tratar de comprender sus preceptos.

Esta perspectiva precisa de una mirada dinámica de doble enfoque, de un movimiento de paneo que debemos ejercer en nuestro actuar como académicos: alejarnos para tener la panorámica de los valores de la racionalidad substantiva y de la reciprocidad, acercarnos para proponer categorías de análisis del cotidiano administrativo, y volvernos a alejarnos para someter a crítica también estas nuevas propuestas, para deconstruirlas y darles nuevos significados; en un permanente ejercicio de paneo que denuncia, anuncia y construye, para volver en movimientos de espiral a generar nuevas propuestas de imaginación organizacional.

Este movimiento de paneo muestra también las varias esferas en el mundo de la administración: la perspectiva que se aleja de lo cotidiano permite pensar, deconstruir lo que está subyacente y proponer nuevos significados desde la perspectiva de la emancipación del ser humano, aportando al crecimiento de la teoría; así como también la mirada que se acerca a la práctica administrativa para proponer criterios de caracterización, aportando al desarrollo de la técnica administrativa. Un ir y venir entre la reciprocidad y la cohesión, sin quedar atrapados en la jaula del control de los dominadores. De los dos enfoques, el que privilegia nuestra mirada es el de la ontología y la epistemología, por sobre la técnica y la metodología.

Una perspectiva típica instrumental de la organización, la ve como inserta en el mercado, en el cual experimenta su trabajo y sus problemas en términos de su propio mundo local, pero no es capaz de comprenderla como parte de un marco de referencia mayor. Por tanto, es preciso que se desarrolle una capacidad de imaginación organizacional que permita entender el verdadero significado de su propia experiencia social. 


\section{La utopía como método de imaginación organizacional}

Ruth Levitas (2010) propone la reconsideración de la utopía como método sociológico, recordando el interesante hecho de la presentación que hiciera H. G. Wells -reconocido autor de La Guerra de los Mundoscuando postulara a su membresía en la Real Sociedad de Sociología. En ella, Wells planteó la utopía como el método distintivo y único de la sociología, en el sentido de que las configuraciones sociales alternativas surgían como fruto del pensamiento utópico en el ensamble de nuevas concepciones de las relaciones de poder y las estructurales sociales. Las organizaciones existen en la sociedad, y por extensión, los autores de este ensayo consideran que las configuraciones y arreglos organizacionales pueden servirse del pensamiento utópico como método para encontrar alternativas nuevas a las predominantes; estas últimas se basan en el cálculo utilitario de consecuencias y en la hegemonía del mercado.

Se trata de constatar que existen otras maneras de pensar acerca de cómo los seres humanos pueden organizarse, y que estas alternativas surgen primero en la mente del estudioso y en el pensamiento utópico. Las utopías clásicas pueden ser vistas como imaginaciones mentales que proponen diferentes enlaces con las principales variables de la organización. Tomás Moro (2004), por ejemplo, escribe sobre las formas alternativas de la democracia, la división del trabajo, la movilidad social, la economía y el intercambio; así como del derecho y la moral, la educación y el control - entre otras cuestiones. Similares abordajes se pueden hacer analizando La República de Platón (1975), obra en la cual, por ejemplo, Sócrates propone mirar al mercado como el espacio en el cual los seres humanos comparten el fruto de su trabajo. De igual suerte, Campanella, Bacon y otros autores de las utopías clásicas, han tratado en sus obras sobre las dimensiones del "espacio-dinámica organizacional", sea viajando a lugares lejanos o transportándose en el tiempo hacia el pasado o el futuro. En estas obras aparecen formas diferentes en las que se puede imaginar los ensambles en los cuales los seres humanos pueden organizarse para trabajar juntos en la búsqueda de objetivos comunes.

Un punto importante del pensamiento utópico, como lo señalara claramente Mannheim (1987), consiste en el hecho de que la utopía, incongruente por naturaleza con la realidad que se vive en un lugar y momento determinados, es fundamentalmente subversiva contra el pensamiento hegemónico o predominante. La esencia de la utopía es la búsqueda de la transformación de lo existente, la formulación de lo inédito realizable, la capacidad anticipadora de posibilidades. El momento en que una utopía se logra, deja de ser utopía, pues su esencia es estar siempre en camino de búsqueda de nuevos horizontes; de lo contrario, se convierte en ideología que trata de mantener el status quo, las relaciones de poder o las estructuras predominantes. ¿No es acaso signo de permanente búsqueda y apertura que Tomás Moro propusiera que en la oración fundamental, en el Credo de los utópicos, se rogara por tener la mente abierta a nuevas posibilidades, a cambiar incluso de religión, si una mejor apareciera en el futuro? (MORO, 2004, p. 305).

En este sentido, la utopía puede verse como una investigación sistemática de principios alternativos de organización (PARKER, 2002). La mayoría de las utopías se basan en la reformulación de las estructuras y dinámicas sociales; en el campo de la administración, esta utopía metodológica permite concebir nuevas formas de organización distintas a la de la hegemonía gerencial del mercado o de la forma empresarial funcionalista. Las reglas explícitas e implícitas necesitan ser deconstruidas y reensambladas, los principios de jerarquía y autoridad decisoria deben ser repensados, los principios de reciprocidad y ética requieren ser valorados, y así sucesivamente. De manera concreta, no creemos que exista solo una manera mejor en la que los seres humanos pueden organizarse.

Marcelo Milano Vieira y Rosimeri Carvalho da Silva (2011), en sus propuestas sobre nuevas posibilidades de estudios de la organización, plantean que es necesario estudiar formas distintas de la empresa y que se precisa definir cómo las conceptualizamos. Así, afirman, se puede extender el objeto de estudio y cruzar las barreras entre las disciplinas para contribuir a la construcción de otros modos posibles de organizar la sociedad. Coinciden con lo que planteamos, en el sentido de que es necesario quitar la técnica del centro de las explicaciones que se dan a las construcciones humanas y adoptar la organización y la producción de organización como un fenómeno social total que trasciende las barreras de las disciplinas. 
La alternativa a la unidimensionalidad o uniformidad es un ethos que permite la autorrealización de los seres humanos y el ejercicio histórico de la racionalidad sustantiva en un mundo de la vida en que el hombre y la república están por sobre el capital y el mercado, los que en la delimitación pierden su presencia hegemónica y tienen más bien una existencia subsidiaria, de apoyo o auxiliar. Las categorías de análisis de nuestra línea de investigación, en el que se inscribe este ensayo, consideran el trabajo como espacio de emancipación del ser humano, la autoridad decisoria como espacio social deliberativo, y la racionalidad substantiva como el principio orientador del sistema social. Con estos lentes pueden analizarse y contrastarse la diversidad de organizaciones existentes en una sociedad particular, con la que están indisolublemente imbricadas en su dinámica histórica.

\section{La imaginación organizacional en Guerreiro Ramos}

La vasta obra de Guerreiro Ramos puede ser abordada desde varias perspectivas; este artículo se aproxima a ella con el objetivo de valorar sus percepciones sobre el fenómeno administrativo, en las que se ha priorizado un examen sociológico e histórico, y sus relaciones de significado con la utopía y la ciencia. Para ello, se plantea en primer lugar el significado de la utopía cómo método de imaginación organizacional y luego el sentido del estatus científico de los estudios organizacionales. En base a estas reflexiones, se analiza la obra de Guerreiro Ramos expresada en sus propuestas de la reducción sociológica, el hombre parentético, la teoría de la delimitación de los sistemas sociales, y la nueva teoría de la administración.

La imaginación sociológica, según Mills (1959), permite que un individuo relacione su propia experiencia personal con las condiciones del contexto mayor de la sociedad. La imaginación organizacional permite comprender la historia y la narrativa de la organización y sus relaciones dentro de la sociedad. En este sentido la organización es una fracción de la sociedad en movimiento. La imaginación organizacional implica mantener una actitud de apertura, y crítica a la vez. Se intenta mostrar en este texto que la reimaginación del "espacio-dinámica organizacional" (MATOS MARTINS, 2011) tiene que ir mano a mano con una exploración de la imaginación como uno de los sitios claves en donde se juegan las agendas organizacionales. La "imaginación" es el nuevo nombre de la utopía. La utopía, en esencia, es la declaración de una organización alternativa.

Imaginamos a la organización tanto como el actuar humano que organiza, como el sistema social por él organizado. Las organizaciones son sistemas sociales en los que sus participantes trabajan para el logro de determinados objetivos y actúan animados por cierto principio orientador. Estas organizaciones están profundamente imbricadas en la trama social y actúan con su propia dinámica inserta en la historia de su sociedad concreta. Por ello, miramos a la organización tanto como un sistema social como una praxis social, tanto como el espacio concreto de trabajo como el actuar que lo configura históricamente. En este sentido, concebimos a las organizaciones como un tejido, en el que cada una de sus tramas contiene fracciones de la sociedad en movimiento.

Esta idea de la organización como fracción de la sociedad en movimiento, en el sentido de que una organización está indisolublemente tejida con la historia y la vida que anima a una sociedad concreta, es un desplazamiento del concepto propuesto originalmente por Santos (1988) para el espacio, que lo concebía como un sistema geográfico-natural-social: "El espacio es (...) un conjunto de formas, cada una de las cuales contiene fracciones de la sociedad en movimiento" (SANTOS, 1988, p. 9)

Desplazando el concepto que Mills planteara hacia el mundo de las organizaciones, se podría expresar que, una organización típica inserta en la corriente predominante del mercado experimenta su trabajo y sus problemas en términos de su propio mundo local, pero no los comprende como parte de un marco de referencia mayor. Por tanto, es preciso que se desarrolle una capacidad -que Guerreiro Ramos llama la reducción sociológica - que permita entender el verdadero significado de su propia experiencia social. 
Cabe preguntarse en este punto si la utopía o imaginación organizacional que destacamos en el pensamiento crítico de Guerreiro Ramos tiene el estatus de ciencia planteados en su obra cumbre "The New Science of Organizations: A reconceptualization of the Wealth of Nations" (1981). El propio título, desde la perspectiva del autor, postula no solamente la idea de una nueva mirada científica al fundo organizacional, sino que tiene grandes pretensiones como para reconceptualizar la obra clásica de Adam Smith y discutir la posición hegemónica del mercado en la sociedad y en la vida humana. ¿Cuál es, entonces, la "ciencia" en la "Nueva Ciencia"? Para aproximarse a las características que definen a la ciencia -y así interpretar la obra de Guerreiro Ramos- se parte de la reconocida propuesta de King, Keohane y Verba (1994), quienes argumentan que la investigación científica tiene cuatro características fundamentales:

1) Su objetivo es hacer inferencias que van más allá de las observaciones particulares que se obtienen, es decir existe la aspiración de emprender en un proceso de generalización, que puede aplicarse en otros contextos similares; estas inferencias pueden ser causales o descriptivas; 2) Los procedimientos son públicos: la investigación científica utiliza métodos y procedimientos que se declaran de manera explícita y extensiva, de forma tal que su confiabilidad puede ser evaluada y contrastada - tal vez replicada - por otros investigadores curiosos; en las ciencias sociales, todos los aspectos de la producción del conocimiento deben darse a conocer de manera expresa y abierta para todos; 3) Las conclusiones son inciertas; por definición, el proceso de inferencia es un proceso imperfecto y que trata con la incertidumbre; debemos convencer de que nuestras estimaciones son razonables, con la humildad reconocer que no son definitivas y están sujetas a nuevas aproximaciones y hallazgos; 4) El contenido es el método; al contrario de lo que generalmente podría suponerse, lo científico no está en la disciplina, sino en el método, en el camino del descubrimiento, que es lo que da unicidad a la ciencia.

Duneier (2012) propone que a estas características de la ciencia, debería agregarse una quinta, particularmente para las ciencias sociales: la reflexividad, esto es, la forma que la participación del investigador influye en los hallazgos, pues todos tienen su propio locus de enunciación -que depende de quién habla, desde dónde y para quién se habla- y las inclinaciones personales tienen una influencia a veces no percibida sobre la actividad investigativa.

En base a este abordaje del estatus científico en las investigaciones sociales, se propone que la obra de Guerreiro Ramos, que él mismo define en la última fase de su pensamiento como "La Nueva Ciencia", es en efecto ciencia, pues tienen los elementos que se han especificado anteriormente como características que distinguen a la ciencia social: su objetivo es la inferencia y la propuesta de un modelo general que es la "para-economía"; sus procedimientos son públicos y explícitos; sus conclusiones permiten avizorar nuevos ensambles organizacionales, aceptando la incertidumbre de sus propuestas como camino de descubrimiento, su método propuesto es el de la reducción sociológica, que lo hemos traducido como imaginación organizacional; y, finalmente, deja claro el particularismo que cada solución implica dependiendo de la perspectiva propia. Es importante, ahora, adentrarse en la esencia de la obra de Guerreiro Ramos, para tomar las ideas claves de la reducción sociológica, el hombre parentético, la teoría de la delimitación de los sistemas sociales y la nueva ciencia de la administración.

En A redução sociológica Guerreiro Ramos (1958) plantea que una comunidad requiere practicar la reducción sociológica para conocer su realidad, sirviéndose de las experiencias de otras, pero centrándose en la realización de su propio proyecto histórico. Con ello, busca impedir la transposición de problemáticas ajenas a la realidad estudiada. La reducción sociológica tiene un triple sentido: a) asimilación crítica de la ciencia foránea; b) resistencia a la masificación de la conducta y a las presiones organizacionales; y c) superación de la ciencia social de los "moldes" en que se encuentra (GUERREIRO RAMOS, 1981).

Las teorías predominantes habían propuesto modelos deterministas, dejando de lado valores particulares y enfatizando las características universales del mundo occidental industrializado. Guerreiro Ramos objetó estas ideas, y argumentó que los países en la periferia no podían pasivamente e ingenuamente, seguir estos ejemplos. De allí que abogó por el desarrollo en Brasil de teorías propias, a través de la "asimilación crítica del patrimonio sociológico extranjero". 
Esta "reducción" se refiere a la asimilación crítica, como un proceso de "destilación" de las ciencias sociales internacionales, para reducirlas al componente que es relevante y útil para cada país. La literatura extranjera debe ser, por tanto, subsidiaria de la realidad latinoamericana (VENTRISS y CANDLER, 2005). Esta propuesta tiene un fuerte influjo de la fenomenología de Husserl (1999) y su propio concepto de reducción fenomenológica: el conocimiento de las esencias a través de la transición de una actitud ingenua a una actitud crítica" "La reducción sociológica es una actitud metodológica que busca aprehender las estructuras fundamentales de los conceptos sociológicos, los fenómenos o los datos, mediante la reducción o la destilación de los presupuestos referenciales, de carácter histórico, de los hechos u objetos de la realidad" (GUERREIRO RAMOS, 1958).

La agenda de la reducción sociológica la fue construyendo Guerreiro Ramos a partir de dos categorías conceptuales, que las propuso al inicio de la década de los cincuenta del siglo pasado: a) la hipercorrección: tendencia de los actores sociales a atribuir una eficiencia directa a las ideas y teorías importadas, sin tomar en cuenta los contextos en los cuales fueron enunciadas y en los que se las aplica; y b) el pragmatismo crítico: característica de los actores sociales que se identifican con el elemento nacional y son sensibles a las condiciones del contexto en el que viven, de cuestionar la ejemplaridad abstracta de las ideas y teorías importadas, sirviéndose de manera oportunista de ellas.

El método de reducción sociológica es una de las grandes contribuciones de Guerreiro Ramos para los estudios críticos en administración. Este método fue revisitado en "The New Science of Organizations" (1981) para demostrar que la teoría organizacional mimetizaba a la sociología, en lo que se refiere al fenómeno de la hipercorrección, es decir, a la asimilación acrítica de ideas y métodos extranjeros. Como consecuencia, se genera una utilización inadecuada de conceptos, como, por ejemplo, el trasplantar ideas de la psicología individual hacia los contextos organizacionales, en lugar de producir definiciones apropiadas que surgen de la psicología social. Este emplazamiento inapropiado de conceptos (uso de los conceptos fuera de su contexto apropiado) se deriva de su obra La Reducción Sociológica. Guerreiro Ramos llama la atención sobre el conflicto entre el pensamiento administrativo visto como universal y el pensamiento administrativo visto desde una perspectiva particular (CANDLER, 2002). En la argumentación respecto a su defensa del particularismo administrativo, Guerreiro Ramos presenta su propuesta sobre la reducción sociológica, como la necesidad de adaptar los conceptos universales a los casos particulares.

La administración pública está profundamente interrelacionada con la sociedad, y críticamente depende del contexto local, regional y nacional. Por tanto, la investigación política es crucial para comprender el contexto (VIEIRA, 2006), y América Latina debe adaptar las lecciones del desarrollo de otras naciones, sobre todo, las de los países desarrollados. Por un lado, Guerreiro Ramos vio la diferencia entre centro-periferia como fundamental, y criticó fuertemente la adopción mecánica de las experiencias del desarrollo de los países centrales. Por otro lado, dentro de la periferia, él destacó el particularismo de cada país latinoamericano (CANDLER, 2002). El desarrollo de un país, por tanto, debe considerarse como un proyecto colectivo, con individualidad histórica, en la que éste pueda ser soberano de su realidad y determinar su futuro por sí mismo.

La preocupación de Guerreiro Ramos por la sociedad contemporánea, se expresa su artículo "Modelos de Homem e Teoría Administrativa" (1972), en el cual propone su concepto antropológico clave, el del "hombre parentético", fundamento del cual deriva su visión de la sociedad multicéntrica y el diseño de sus sistemas sociales (AZEVEDO y ALBERNAZ, 2006). También aquí hay una fuerte influencia de Husserl (1999), quien planteó que el conocimiento de las esencias sólo es posible a través de la "parentización", esto es, poner entre paréntesis lo que se supone como ya sabido para llegar a las esencias de las cosas. Metafóricamente, así como Platón basó su visión de la educación y desarrollo del ser humano, su paideia, comparándolo con la ciudad ideal en la República, Guerreiro Ramos plantea revisar la trayectoria de la teoría

\footnotetext{
${ }^{4}$ La reducción fenomenológica, según Schmitt (1959), implica un cambio de actitud hacia un pensamiento crítico, a través de poner entre paréntesis y en suspensión lo que se da por hecho, para reflexionar sobre las esencias subyacentes.
} 
social y administrativa usando como punto de referencia tres modelos de ser humano: el hombre operacional, el hombre reactivo y el hombre parentético.

En el inicio de la teoría administrativa de Taylor, se considera al ser humano como un hombre operacional, como un recurso a ser maximizado en términos de un producto medible y como una extensión de la máquina. El hombre operacional es un ser pasivo que debe ser programado para maximizar la producción; un ser calculador motivado por recompensas materiales, aislado e independiente de otros.

Los "humanistas" pretendieron mejorar la teoría organizacional planteando un ser humano alternativo, con una visión más sofisticada de la naturaleza de la motivación humana, con una visión de la organización como un sistema abierto y la consideración de los valores, los sentimientos y las actitudes en el proceso productivo. Este ser humano, según Guerreiro Ramos, puede ser llamado como hombre reactivo. El hombre reactivo es un ser que reacciona, que se comporta de acuerdo a los estímulos funcionales a la organización, que se ajusta a los contextos del trabajo y no a su realización personal.

Como una emancipación del hombre operacional o reactivo, conformista, aprisionado en su inmediatismo, Guerreiro Ramos propone el hombre parentético; un ser en "suspensión", que está "entre paréntesis", con conciencia crítica sobre su realidad cotidiana. Esta conciencia crítica le permite poner entre paréntesis las creencias divulgadas o las normas impuestas, para reflexionar críticamente sobre ellas y, por tanto, ejercer su libertad. Además, el hombre parentético se compromete éticamente con la vida social, dejando de lado un relativismo irreflexivo. De esta forma, "la actitud parentética se define como la capacidad psicológica del individuo para separarse de sus circunstancias internas y externas. Los hombres parentéticos prosperan cuando termina (...) la ingenuidad social” (GUERREIRO RAMOS, 1972).

Frente a un hombre organizacional, restringido por su sujeción al mercado, Guerreiro plantea un hombre parentético con verdadera participación social. "Es por eso que hoy no basta administrar las organizaciones, es necesario administrar la sociedad toda" (GUERREIRO RAMOS, 1972). Este es uno de los puntos centrales que permea la obra de Guerreiro Ramos: un humanismo radical -en el sentido de raíz- que percibe el potencial de destrucción que se encuentra en el fenómeno organizacional moderno, sobre todo en las formas de organización utilitaristas y dominadas por el ethos del mercado.

El planteamiento básico de Alberto Guerreiro Ramos (1976) es la de constituir varios escenarios y enclaves en la sociedad, en los cuales el ser humano pueda realizarse a sí mismo. Esta línea de pensamiento intenta responder a la problemática planteada por Hanna Arendt (1974), respecto a constituir "lugares adecuados" que permitiesen al ser humano contemporáneo el ejercicio de actividades dirigidas a su realización. Por ello, Guerreiro Ramos propone una formulación tipológica de seres humanos y los correspondientes escenarios sociales que puedan servir para el diseño organizacional.

El mercado es sólo un enclave más, ya no es el centro hegemónico. De esta manera se configura una propuesta de carácter multidimensional, con diversidad de posibilidades de realización individual y de consecución de logros sociales. La noción de delimitación social implica que: a) la sociedad se compone de múltiples dominios, dentro de los cuales los sujetos se asocian en diversas actividades; y b) un gobierno social que formula e implementa políticas para distribuir los recursos y tomar las decisiones necesarias para la interacción entre los diversos dominios o enclaves sociales.

La crítica de la razón instrumental, centrada en el mercado, propone la razón sustantiva, subjetiva, centrada en la realización del ser humano y en el entendimiento (SERVA, 1997; TENÓRIO, 2004). Las organizaciones actuales no son el escenario apropiado para esta autorealización, pues pretenden la total inclusión de las personas en sus límites: la economía. El mercado es la fuerza que modela la sociedad como un todo. La naturaleza humana se la entiende dentro del mercado, el hombre, de acuerdo a su (des)empleo; la comunicación, como instrumental (maximiza el lucro), como política cognitiva que subordina y engaña. Una sociedad centrada en el mercado provoca degradación de la calidad de vida, inseguridad psicológica, contaminación, desperdicio de los recursos naturales del planeta, a más de producir una teoría organizacional incapaz de construir espacios sociales gratificantes para el individuo y la comunidad. 
Guerreiro Ramos (1976) hace una distinción entre comportamiento y acción para esclarecer la teoría organizacional convencional. El comportamiento es una forma de conducta basada en la racionalidad funcional, desprovista de contenido ético de validez general y dictado por imperativos exteriores; la acción, en cambio, viene de la conducta ética de un agente que delibera sobre las cosas porque está consciente de sus finalidades. El problema radica en que el síndrome comportamentalista ha plagado la teoría organizacional convencional. Los rasgos de este síndrome son: a) la fluidez de la individualidad, que está relacionado con una relatividad moral, que no considera nada como bueno o malo en sí mismo, y con una conducta calculadora, guiada por las reglas objetivas de convivencia; b) el perspectivismo, que incluye la comprensión de que las conductas son afectadas por una perspectiva, llevando a que cada individuo calcule la visión del otro, para manipularlo; c) el formalismo, que contempla un conjunto de conductas amoldadas a los imperativos externos, a los cuales recurre el individuo para superar la alienación causada por el relativismo moral y el egocentrismo; y d) el operacionalismo, que recurre a los métodos de las ciencias naturales, de características matemáticas e inspiración positivista, como forma de validad y verificar el conocimiento, adscribiéndose a una orientación controladora del mundo e infiriendo que las cosas son resultados de causas eficientes, toda vez que interpreta el mundo como una sucesión de antecedentes y consecuentes.

La formulación teórica de Guerreiro Ramos tiene una fuerte influencia de los estudios de Karl Polanyi (1944), quien, como coordinador de un grupo interdisciplinario en Columbia University, estableció la concepción sustantiva de la economía en la década de los cuarenta del siglo pasado. Polanyi contestaba la idea de que hubiese que tomar la razón instrumental como punto de partida para el análisis de todas y cada una de los sistemas sociales y económicos. El defendía la tesis de que la economía debía ser analizada como un proceso social, esto es, inserto en la configuración propia de cada sociedad históricamente definida. Por tanto, la racionalidad instrumental o de mercado no serviría como categoría de análisis para todos los sistemas económicos. En base a esta demarcación, Polanyi acuñó la expresión de concepción sustantiva, la cual concentra el interés sobre "los valores, la motivación y la política". De allí que esta concepción de Polanyi va a constituir una de las principales fuentes de inspiración de Guerreiro Ramos, y de la cual aprovechó el terminología sustantiva y en la que inspiró su planteamiento de la teoría de la delimitación de los sistemas sociales.

Frente a la visión instrumental, centrada en el mercado, orientada a la maximización de la productividad, Guerreiro Ramos plantea un modelo de razón sustantiva, para satisfacer las necesidades de realización personal en múltiples escenarios sociales. En contra de esta visión unidimensional, centrada en el mercado y la economía, plantea una ecología global de existencia humana (BOEIRA, 2002). En esta consideración de la ecología humana y de respeto al ambiente, Guerreiro Ramos fue un visionario, y es uno de los puntos de encuentro con el principio de Sumaq Kawsay (SALGADO, 2010), que ve al ambiente como la Pachamama, la madre que proporciona y protege la vida.

La utopía, como investigación sistemática de principios alternativos de organización, se encuentra expresada en el libro "The New Science of Organizations: A reconceptualization of the Wealth of Nations", publicado en 1981 en Estados Unidos. Guerreiro Ramos hace una crítica de la razón instrumental proponiendo la centralidad del ser humano frente a las organizaciones; luego discute los presupuestos o puntos débiles de la teoría organizacional convencional, para proponer un abordaje sustantivo de las organizaciones. Plantea, entonces, las directrices de la nueva teoría de las organizaciones, en base a un modelo multicéntrico de la sociedad, es decir, una sociedad compuesta por una variedad de organizaciones y de relaciones capaces de atender las diversas necesidades humanas.

Desde el primer capítulo de su libro, Guerreiro Ramos (1981) plantea que la teoría organizacional predominante es ingenua (naive), porque está determinada en base de la racionalidad instrumental, inherente a la ciencia social occidental. Esta ingenuidad ha permitido que la racionalidad instrumental consiga sus objetivos prácticos. Estos procesos han sido unidimensionales y han desfigurado la vida humana en sociedad, por lo que se cuestiona la ética administrativa predominante (CANDLER y VENTRISS, 2006). La formulación multidimensional propuesta por Guerreiro Ramos permite buscar diversas formas organizacionales que tiendan a conjugar los objetivos de realización de las personas con una óptima 
asignación de recursos para la producción de bienes y servicios. Se toma en cuenta tanto actividades remuneradas como no remuneradas, escenarios formales como informales.

Para conceptualizar su visión multidimensional, Guerreiro Ramos parte de la forma predominante y la denomina economía. Toma entonces las raíces griegas de la palabra: оэкко-vopía para proponer otros tipos

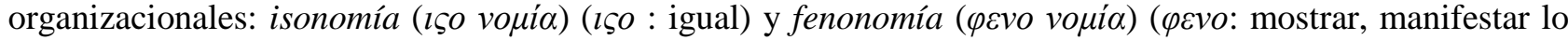
oculto, crear). Esta construcción lingüística es muy interesante: оюкко vopí $\alpha$ se entiende normalmente como administración, como el "cuidado de la casa". Es importante notar que el significado original de vouía es el de cuidar con ternura, y que se lo aplicaba a las mujeres a cargo de los bebés. El significado de administración, en griego, es pues el de cuidar con amor el bien común. Este significado corresponde plenamente con el sentido original de la palabra latina administrāre, que significa servir, y se forma de $a d$ min (min significa mano, y ad es una preposición que indica movimiento), es decir el símbolo de dar la mano, de tender la mano.

El significado original de cuidado y servicio, se ha cambiado por un sentido de control y ahorro. En el Diccionario de la Real Academia de la Lengua Española (, se tienen al menos cuatro acepciones del término economía en este último sentido: a) Contención o adecuada distribución de recursos materiales o expresivos. b) Ahorro de trabajo, tiempo o de otros bienes o servicios; c) Ahorros mantenidos en reserva; d) Reducción de gastos anunciados o previstos.

Guerreiro Ramos llama la atención sobre el hecho de que los teóricos organizacionales convencionales han enfocado su interés solamente en las organizaciones económicas, dejando en segundo plano el análisis sistemático de la variedad de sistemas sociales presentes en el espacio social. Considerando que las organizaciones económicas son apenas un caso particular de diversos tipos de sistemas, deberían limitarse a sus objetivos sin invadir el espacio vital humano. El comportamiento administrativo no puede ser considerado lo central en la vida humana, pues es incompatible con el desarrollo a plenitud de la persona. Por ello, es necesario crear otras oportunidades de realización individual en otros espacios sociales, evitando una "superorganización" que transforma la sociedad en un universo en el cual el individuo solamente es un actor de un papel determinado, sin espacio ni tiempo para una vida personal creativa.

Esta diversidad de tipos de organización puede ir desde un ambientes organizacional con gran normatividad (economías) hasta aquel con ausencia de normas (anomías), debiendo recordarse que los límites de las dimensiones consideradas son referenciales, en un continuum donde es posible encontrar diversas formas organizativas entre sus opuestos; en donde una diversidad de organizaciones pueden ser socialmente construidas. Uno de los objetivos del paradigma para-económico es una equilibrada asignación de recursos, tomando en cuenta la realidad de cada entorno. Así, la existencia de una economía diversa en un país puede constituir una ventaja en lugar de un inconveniente, que permite la coexistencia de comunidades del llamado tercer sector con otras formas de organización.

En uno de los escenarios de las categorías delimitadoras de la dimensión individual/comunitaria de organización, está el tipo de organización denominada isonomía, en la cual todos los miembros son iguales, en derechos y deberes, y les permite una realización personal; es autogratificante, sus actividades corresponden a vocaciones, más que a empleos, y en donde la toma de decisiones es consensual, por ejemplo en empresas comunitarias y cooperativas, entre otras. En otro parte está la fenonomía, donde existen sistemas sociales más o menos estables, de tamaño pequeño en número, con fines u objetivos compartidos. Son ejemplos de fenonomías: las comunidades científicas, los grupos de investigadores, las comunas de artistas, grupos ecológicos, y otros similares. De igual manera que en el caso anterior, sus miembros logran una realización personal, son autogratificantes y sus actividades corresponden a vocaciones antes que a empleos. La tabla 1 resume este modelo para-económico así planteado. 
Tabla 1

Modelo para-económico: Posibilidades de la utopía como imaginación organizacional

\begin{tabular}{|l|l|l|l|l|l|}
\hline & Tecnología & Tamaño & Sistema & Espacio & Tiempo \\
\hline $\begin{array}{l}\text { Econnitivo } \\
\text { (burocracia) }\end{array}$ & $\begin{array}{l}\text { Alto grado de } \\
\text { organización }\end{array}$ & $\begin{array}{l}\text { Grande- } \\
\text { mediano }\end{array}$ & Funcional & $\begin{array}{l}\text { Socio-fugaz } \\
\text { (centrífugo) }\end{array}$ & $\begin{array}{l}\text { Serial } \\
\text { (Cronos) }\end{array}$ \\
\hline $\begin{array}{l}\text { Isonomía } \\
\text { (igualdad) }\end{array}$ & $\begin{array}{l}\text { Orientada a } \\
\text { la realización } \\
\text { de los seres } \\
\text { humanos }\end{array}$ & $\begin{array}{l}\text { Moderado } \\
\text { (Con un } \\
\text { mínimo y } \\
\text { un máximo) }\end{array}$ & Político & $\begin{array}{l}\text { Socio- } \\
\text { aproximado } \\
\text { (centrípeto) }\end{array}$ & Convivencial \\
\hline $\begin{array}{l}\text { Fenonomía } \\
\text { (creatividad) }\end{array}$ & $\begin{array}{l}\text { Orientada a } \\
\text { la innovación }\end{array}$ & Pequeño & Personal & $\begin{array}{l}\text { Socio- } \\
\text { aproximado }\end{array}$ & $\begin{array}{l}\text { Salto (Kairós) } \\
\text { oportunidad }\end{array}$ \\
\hline
\end{tabular}

Fuente: Basado en The New Science of Organizations (GUERREIRO RAMOS, 1981).

Para confluir, el Principio de los Recursos Adecuados (o de la diversidad de requisitos) establece que, para una variedad de sistemas sociales, éstos puedan seleccionar sus propios requisitos de planificación, con el objetivo de lograr que sus miembros accedan a condiciones de realización personal y de satisfacción de necesidades. La adecuada selección de alternativas, entre las principales dimensiones de los sistemas sociales, permite el diseño apropiado de dichos sistemas. Guerreiro Ramos propone cinco dimensiones: la tecnología, el tamaño, el sistema cognitivo, la distribución del tiempo, y la organización del espacio, que permitirán lograr mejores condiciones de vida para sus integrantes.

Reflexionemos ahora sobre los elementos de utopía como imaginación organizacional que se encuentran en el modelo para-económico de Guerreiro Ramos. La diversidad de alternativas por él planteadas tienen que ver, en primer lugar, con el tamaño o escala de la organización: Desde Weber (2005) a las actuales protestas antiglobalización (CASTELLS, 2012), una de las críticas principales se refiere a que la organización se está volviendo tan grande que deja de ser humana (KORTEN, 1995; MONBIOT, 2000). Esta dimensión gigante de las organizaciones tienen serias consecuencias como el gran poder e influencia que les permite pasar por alto sus límites; su cobertura y jerarquía hacen que la autoridad decisoria sea concentrada en pocas personas a las que no se pide cuenta por los efectos de sus decisiones y además no favorecen la diversidad de modos de organización pues las alternativas son pocas y tienden a la homogeneización.

Guerreiro Ramos plantea también otras dimensiones en los que la acción de organización puede ser reimaginada. Al plantear las formas ideales de fenonomías e isonomías, llama la atención sobre las posibilidades que se encuentran en las organizaciones en las que la creatividad y la reciprocidad son esenciales para su generación y persistencia. De este modo propone una gama de alternativas que discuten la visión de imperativos inmutables o de contingencias del entorno o suposiciones de corta visión sobre el control, autoridad decisoria, relaciones interpersonales, recompensas y sanciones.

La imaginación organizacional propuesta por Guerreiro Ramos destaca el actuar racional sustantivo orientado por dos dimensiones: una dimensión individual, que se refiere a la autorrealización, comprendida como concretización de las potencialidades y la satisfacción; y una dimensión grupal, que se refiere al entendimiento, y a la responsabilidad y satisfacción sociales. El actuar racional sustantivo se contrapone a la acción instrumental, cuyos elementos constitutivos son la acción basada en el cálculo, orientada para el 
alcance de metas técnicas o de finalidades ligadas a intereses económicos o de poder social, a través de la maximización de los recursos disponibles.

\section{Conclusiones}

El pensamiento de Guerreiro Ramos, que para el efecto de este artículo se ha sintetizado en las propuestas de: la reducción sociológica, el hombre parentético, la teoría de la delimitación de los sistemas sociales y la nueva ciencia de las organizaciones, puede comprenderse a la luz de los planteamientos respecto a la utopía como método de imaginación organizacional o la investigación sistemática de principios alternativos al ethos del mercado. La delimitación es un intento sistemático para superar el continuo proceso predominante de uniformizar tanto al ser humano como a la vida colectiva.

La unidimensionalización, el proceso específico de socialización por el cual el ser humano internaliza profundamente el ethos del mercado y actúa de manera tal como si este ethos fuera la dimensión normativa absoluta del espectro total - el único color del arco iris - de las relaciones interpersonales, es contestada con la propuesta de diferentes escenarios, formas y dinámicas para rearticular los principios de la administración y sus diferentes dimensiones y dinámicas. El campo organizacional es un complejo multidimensional de relaciones entre los sujetos que lo conforman. Se puede intentar analizar ese complejo, descomponiéndolo en algunas dimensiones o variables que se consideren como significativas para su comprensión. Sin embargo, se debe tener en cuenta que sólo se lo hace con este propósito, pues la dinámica del campo organizacional responde a la convergencia de las relaciones entre todas sus variables y no solamente de alguna de ellas.

La alternativa a la unidimensionalidad o uniformidad es un ethos que permite la autorrealización de los seres humanos y el ejercicio histórico de la racionalidad sustantiva en un mundo de la vida en que el hombre y la república están por sobre el capital y el mercado, los que en la delimitación pierden su presencia hegemónica y tienen más bien una existencia subsidiaria, de apoyo o auxiliar. Las categorías de análisis de nuestra línea de investigación, en el que se inscribe este ensayo, consideran el trabajo como espacio de emancipación del ser humano, la autoridad decisoria como espacio social deliberativo, y la racionalidad sustantiva como el principio orientador del sistema social. Con estos lentes pueden analizarse y contrastarse la diversidad de organizaciones existentes en una sociedad particular, con la que están indisolublemente imbricadas en su dinámica histórica.

Para comprender las organizaciones es necesario entender a la sociedad en la que actúan. Por ello, el ensamble de las variables del campo organizacional requiere verse tanto desde una perspectiva societaria macro -las ciudades y espacios virtuales en los que actúan- así como desde una perspectiva organizacional micro. Imaginamos a la organización tanto como el actuar humano que organiza, como el sistema social por él organizado. Las organizaciones son sistemas sociales en los que sus participantes trabajan para el logro de determinados objetivos y actúan animados por cierto principio orientador. Estas organizaciones están profundamente imbricadas en la trama social y actúan con su propia dinámica inserta en la historia de su sociedad concreta. Por ello, miramos a la organización tanto como un sistema social como una praxis social, tanto como el espacio concreto de trabajo como el actuar que lo configura históricamente. En este sentido, concebimos a las organizaciones como un tejido, en el que cada una de sus tramas contiene fracciones de la sociedad en movimiento.

El mercado en la para-economía debe ser regulado de forma de no dañar los enclaves isonómicos y fenonómicos que van surgiendo, como serían los sistemas mutuales, cooperativos, comunales, o fábricas gestionadas por sus dueños-trabajadores, que son el embrión de un tipo de organizaciones, que propician la diversidad y la sustentabilidad. Los sistemas auto sustentados son complementarios a los establecidos en las economías actuales y su interrelación los fortalece. 
Se ha considerado el campo organizacional como un ensamble dinámico que ocurre en un contexto societario macro - tanto configurador del campo organizacional como configurado a su vez por éste - y que puede verse de manera multidimensional combinando las diferentes formas de comprender el espacio, el tiempo, la tecnología y el entorno simbólico. La sociedad y los campos organizacionales están en constante movimiento y acción recíproca, y las variables que hemos analizado se desplazan e influyen mutuamente en un complejo indisoluble, pues hay diversos ensambles organizacionales posibles e imaginables, que pueden y deben ir cambiando en su dinámica particular e histórica, en la búsqueda de la razón substantiva y de la realización plena del ser humano.

La perspectiva crítica en la administración precisa de una mirada dinámica de doble enfoque, de un movimiento de paneo que debemos ejercer en nuestro actuar como académicos: alejarnos para tener la panorámica de los valores de la racionalidad sustantiva, acercarnos para proponer categorías de análisis del cotidiano administrativo. La perspectiva de la utopía nos impulsa para volver a alejarnos y someter a crítica también estas nuevas propuestas, para deconstruirlas y darles nuevos significados; en un permanente ejercicio de paneo que denuncia, anuncia y construye; para volver, en movimientos de espiral, a generar nuevas propuestas de imaginación organizacional.

\section{Referencias}

AKTOUF, O. La metodología de las ciencias sociales y el enfoque cualitativo en las organizaciones. Cali: Universidad del Valle, 2001.

ALVESSON, M. Critical Theory and Posmodernism Approaches to Organizational Studies. En: CLEGG, S. R. et al. (Eds.). The SAGE Handbook of Organization Studies. Second Edition. London: SAGE, 2006. 255-283 p.

ALVESSON, M.; BRIDGMAN, T.; WILlMOT, H. The Oxford Handbook of Critical Management Studies. New York: Oxford University Press, 2001.

ALVESSON, M.; DEETZ, S. Critical Theory and Postmodernism: Approaches to Organizational Studies. En C. Grey; H. Willmott (Edits.). Critical Management Studies. A Reader. New York: Oxford University Press, 2005. 60-106 p.

ALVESSON, M.; WILLMOT, H. Critical Theory and Management Studies: An Introduction. En: ALVESSON, M.; WILLMOTT, H. (Eds.). Critical Management Studies. London: Sage, 1992.

ANDREWS, C. Revisiting Guerreiro Ramo's The New Science of Organizations Through Habermasian Lenses: A Critical Tribute. Praxis, v. 22, n. 2, p. 246-272, 2000.

ARENDT, H. La Condición Humana. Barcelona: Seix Barral, 1974.

AZEVEDO, A.; ALBERNAZ, R. Alberto Guerreiro Ramos's Anthropological Approach to the Social Scientes: The Parenthetical Man. Administrative Theory \& Praxis, v. 28, n. 4, p. 501-521, 2006.

BOIERA, S. L. Ecología Política: Guerreiro Ramos e Frit Jof Capra. Ambiente \& Sociedade, v. 5, n. 10, p. 1-21, 2002.

BURRELl, G.; MORGAN, G. Sociological Paradigms and Organisational Analysis. London: Heinemann Educational Books Ltd, 1979.

CANDLER, G. G. Particularims versus Universalism in the Brazilian Public Administration Literature. Public Administration Review, v. 62, n. 3, p. 298-306, 2002.

CANDLER, G. G.; VENTRISS, C. Symposium - The Destiny of a Theory: Beyond the New Science of Organizactions. Introduction to the Symposium: Why Guerreiro. Administrative Theory \& Praxis, v. 28, n. 4, p. 495-500, 2006.

CASTELLS, M. Networks of Outrage and Hope: Social Movements in the Internet Age. Polity Press, 2012. 
CHANLAT, J.-F. Sciences sociales et management. Paris: Éditions Eska, 1998.

DUNEIER, M. Introduction to Sociology. Online MOOC. Coursera. Princeton University, 2012.

FARIA, J. H. Consciência crítica com ciência idealista: paradoxos da redução sociológica na fenomenologia de Guerreiro Ramos. Cadernos EBAPE.BR, v. 7, n. 3, p. 419-446, 2009.

GUERREIRO RAMOS, A. A redução sociológica, en Heidemann, F. (2001) Nota Bibliográfica de a "Modelos de Homem e Teoria Administrativa". Caderno de Ciencias Sociais Aplicadas, PUC-PR/Mestrado en Administração, n. 3, dez. 2001, [1958].

GUERREIRO RAMOS, A. A redução sociológica. Rio de Janeiro: Tempo Brasileiro, 1965.

GUERREIRO RAMOS, A. Modelos de Homem e Teoría Administrativa. Public Administration Review, v. 32, n. 3 , p. 241-246, 1972.

GUERREIRO RAMOS, A. Theory of Social Systems Delineation: A Preliminary Statement. Administration and Society, v. 8, n. 2, p. 249-271, 1976.

GUERREIRO RAMOS, A. The New Science of Organizations. A Reconceptualization of the Wealth of Nations. Toronto: University of Toronto Press, 1981.

HABERMAS, J. El Discurso Filosófico de la Modernidad. Madrid: Taurus, 1989.

HUSSERL, E. Ideas Relativas A Una Fenomenologia Pura. México D. F.: Fondo de Cultura Económica, 1999.

KAYTAL, S. Critical Management Studies: Perspectives on Information System. New Delhi: Global India Publications Pvt Ltd, 2009

KING, G.; KEOHANE, R.; VERBA, S. Designing Social Inquiry: Scientific inference in qualitative reasearch. Princeton. Princeton University Press, 1994.

KORTEN, D. When Corporations Rule the World. West Hartford, Ct: Kumarian Press, 1995.

LEVITAS, R. Back to the future: Wells, sociology, utopia and method. The Sociological Review, v. 58, n. 4, p. 530547,2010

LYOTARD, J.-F. La condición postmoderna (8va. ed.). Madrid: Cátedra, 1984.

MANNHEIM, K. Ideología y Utopía. Introducción a la sociología del conocimiento. Traducción de Salvador Echavarría. México: Fondo de Cultura Económica, 1987.

MATOS MARTINS, P. E. O Espaço-Dinâmica Organizacional em Perspectiva Histórica. Instituto Ricardo Brennan. Recife: Instituto Ricardo Brennan, 2011.

MILLS, C. W. The Sociological Imagination. Harmondsworth: Penguin, 1959.

MONBIOT, G. Captive State: The Corporate Takeover of Britain. London: Macmillan, 2000.

MORO, T. Utopía. Buenos Aires: Longseller. Clásicos de Siempre. Grandes Filósofos, 2004.

PARKER, M. Utopia and Organization. Blackwell Publishing, 2002.

PLATÓN. La República. Barcelona: Editorial Vosgos. Colección Grandes Maestros, 1975.

POLANYI, K. The Great Transformation: The Political Origin of Our Time. Boston: Beacon Press, 1944. 
SAAVEDRA MAYORGA, J. J. Descubriendo el lado oscuro de la gestión: los Critical Management Studies o una nueva forma de abordar los fenómenos organizacionales. Revista Facultad de Ciencias Económicas: Investigación y Reflexión, v. 17, n. 2 p. 45-60, 2009.

SALGADO, F. Sumaq Kawsay: The birth of a notion? Cadernos EBAPE.BR, v. 8, n. 2, p. 198-208, 2010.

SANTOS, M. Metamorfoses do espaço habitado, fundamentos teórico e metodológico da geografia. São Paulo: Hucitec, 1988.

SCHMITT, R. Husserl's transcendental-phenomenological reduction. Philosophy and Phenomenological Research, v. 20, n. 2, p. 238-245, 1959.

SERVA, M. A racionalidade substantiva demonstrada na prática administrativa. Revista de administração de empresas, v. 37, n. 2, p. 18-30, 1997.

TENÓRIO, F. Tem Razão a administração? Cap VI: [Neo]Tecnócratas ou [Neo]Bobos? Eis a Questao. Ijuí: Editora Unijuí de Universidade Regional do Noroeste do Estado do Rio Grande do Sul, 2004.

TENÓRIO, F. Las teorías organizacionales bajo el orden y el progreso. O como los muertos comandan los vivos. PEGS/EBAPE/FGV. Primer Semestre, 2006.

TENÓRIO, F. La mirada de Janus a la Administración. ¿Pero cuál mirada? PEGS/EBAPE/FGV. Primer Semestre, 2010.

VENTRISS, C.; CANDLER G. G. Alberto Guerreiro Ramos, 20 Years Later: A New Science still Unrealized in an Era of Public Cynicism and Theoretical Ambivalence. Public Administration Review, v. 65, n. 3, p. 347-359, 2005.

VIEIRA, M. M. Por uma boa pesquisa (qualitativa) em adminstracao. En: VIEIRA, M. M.; ZOUAIN, D. M. Pesquisa qualitativa em administração. Rio de Janeiro: Editora FGV, 2007. 13-28 p.

VIEIRA, M. M.; CALDAS, M. P. Teoria crítica e pós-modernismo: principais alternativas à hegemonia funcionalista. Rev. adm. empres. v. 46, n. 1, p. 59-70, 2006.

VIEIRA, M. M.; DA SILVA, R. Eight propositions towards new possibilities of studying organizing and organizations. Brazilian Administration Review, Curitiba, v. 8, n. 4, p. 454-467, 2011.

WEBER, M. Economia y Sociedad. Barcelona: Areté, 2005. 